\title{
A Framework for Physical Health Improvement using Wireless Sensor Networks and Gaming
}

\author{
Paul Fergus, Kashif Kifayat, Simon Cooper, Madjid Merabti, Abdennour El Rhalibi \\ Networked Appliances Laboratory, \\ School of Computing and Mathematical Sciences \\ Liverpool John Moores University, \\ Byrom Street, Liverpool L3 3AF, UK. \\ Email: \{P.Fergus, K.Kifayat\}@ljmu.ac.uk, S.Cooper@2003.ljmu.ac.uk, \{M.Merabti, A.Elrhalibi\}@ljmu.ac.uk
}

\begin{abstract}
Wireless Sensor Networks (WSNs) is a recent technological advancement in Micro Electro Mechanical Systems (MEMS). It has a large number of applications in the health sector, for example, the telemonitoring of human psychophysical data, tracking and drug administration in hospitals. In this paper we present a novel framework using body area WSNs and gaming to improve a patient's physical health. The proposed framework has three main components, the body area WSN, the game, and the data acquisition manager. Using the WSN on the patient's body allows real time motion and medical data to be collected. This information is then filtered and used inside the gaming environment to control the patient's avatar. This data also provides a level adjustment mechanism to change gaming parameters according to the medical status of the patient. Whilst the patient interacts within the gaming environment data is regularly collected from body sensor nodes and stored inside the data store. A neck physiotherapy case study is presented to illustrate the applicability of our approach.
\end{abstract}

Keywords - Physiotherapy; Telemonitoring; Telemedicine; Wireless Sensor Networks; Body Area Networks; Games

\section{INTRODUCTION}

New technologies are invented according to the needs of users in order to bring more flexibility to their daily lives. The history of telemedicine is a good example of how communication and information technology can deliver more efficient and flexible clinical care on a day-to-day basis.

Enhancing the obvious benefits this brings, Wireless Sensor Networks (WSNs) promises to offer more. WSNs are a newly developed networking technology consisting of multifunctional sensor nodes that are small in size and that communicate over short distances. Sensor nodes incorporate properties for sensing environments, data processing and communication with other sensors and are typically characterised by limited power supplies, low bandwidth, small memory sizes and limited energy. This technology can allow long-term, unobtrusive, ambulatory health monitoring with instantaneous feedback to the user about the current health status and real-time or near real-time updates of the user's medical records anytime and anywhere. For example, this could be performed in a primary care setting, i.e. the home, as illustrated in Figure 1.

This is seen as crucial in many instances were patients require continuous monitoring. For example, vascular, heart and autoimmune patients fall into such a category of patient were continuous and regular physical therapy is required to improve their physical health. In all these diseases recovery is an ongoing process. The statistical information for such patents in the UK shows that stroke is the biggest cause of disability. About 110,000 people each year experience a first stroke, and a further 30,000 go onto have a further stroke. More than $75 \%$ of these patients require multi-disciplinary assessments and appropriate rehabilitative treatments after they are discharged from the hospital [1-4]. One of the main reasons for physical therapy is to enable patients to regain, as high as possible, functional movement. Therapy for this type of patient has increased and put a significant strain on community healthcare services.

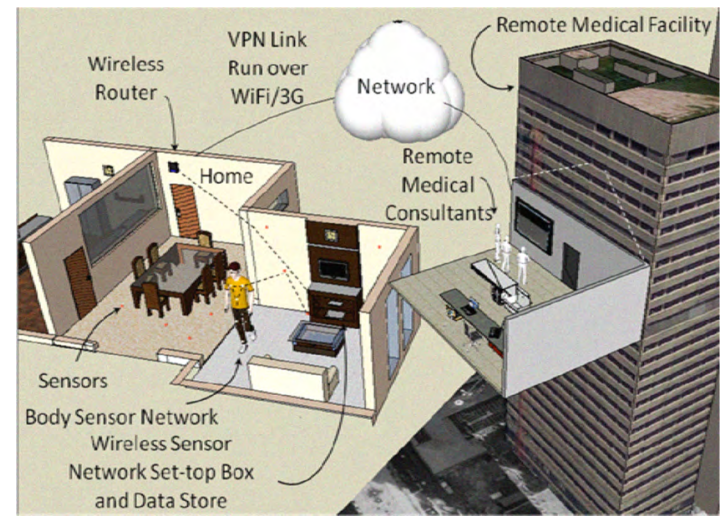

Fig. 1 Healthcare monitoring in the home

Therefore training aids or intelligent systems are required to provide and support rehabilitation for such patients in the home and in medical installations. Consequently, such systems can be used as complementary tools. Furthermore if these systems integrate into a telemedicine system, these system can even alert medical personnel when life-threatening changes occur [5].

In this paper we present a design and implementation of a novel framework for physical health improvement using body area WSNs and gaming. The proposed system can be used inhome (as shown in Figure 1), and data can be communicated anywhere and anytime. This will allow in-community healthcare centres to readily receive this data and react to it. This system is cost effective and provides better long term rehabilitation services to different kinds of patient using fewer resources. We believe this system will also help medical research to investigate different 
diseases by using patient medical data.

The remaining sections of this paper are composed as follows: Section II describes current work. In Section III, we describe our proposed framework architecture before discussing our implementation in Section IV. In Section V we provide our evaluation before concluding the paper in Section VI.

\section{BACKGROUND}

An area that is promising to revolutionise how primary healthcare is administered is Telehealth. With increasing demands to reduce costs the use of technology is being explored, particularly in the area of periodic assessment. At its simplest level such systems are being used to contact carers when alarm conditions are raised [6]. Looking further ahead research is investigating how technology can be used for the early detection of illnesses through the real-time monitoring and extrapolation of physiological data, such as blood pressure, blood glucose levels, and day-to-day weight monitoring amongst other markers [7].

Utilising ubiquitous communications such monitoring can easily be applied within a home environment. For example, patients with asthma can be assessed on spirometry self-testing through the exchange of testing data and clinical recommendations between patients and clinicians [8]. In remote areas patients can form close links with medical facilities through the use of communications, and this has proven to help detect anomalies in such patients in real-time, e.g. blood glucose levels, electrocardiogram readings, including low blood oxygen levels [9].

Current research shows that a great deal of work has been done and in the remainder of this paper we draw from the advances made to create a generic and scalable medical healthcare framework for physiological, kinematics and kinetic analysis using ubiquitous communications and wireless devices.

\section{FRAMEWORK ARCHITECTURE}

The proposed framework has three major components: the body area WSN, the gaming environment, and the data acquisition manager as shown in Figure 2.

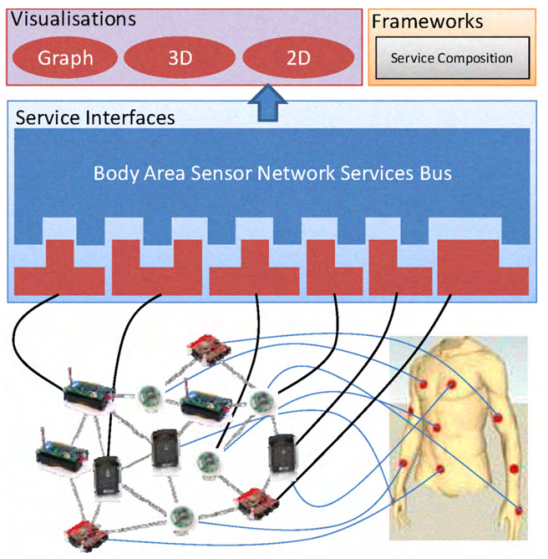

Fig. 2 Illustration of the framework model

The framework utilises a message-based approach for resource discovery and interaction. Functionality is abstracted as services, which are fully extensible. The base discovery tool utilises keyword matching, however this could be extended to include semantic discovery. An overlay built on peer-to-peer technologies allows body area WSNs to be created on the fly. Sensors use peer-to-peer techniques to automatically integrate themselves within the network devoid of any third-party intervention. This is made easy with widely investigated protocols such as Bluetooth, Zigbee (802.15.4) and 802.11.We describe these components in the following subsections.

\section{A. Body Area Wireless Sensor Networks (Component one)}

There are different types of sensor nodes used to establish the body area WSN as shown in Figure 3 (motion sensors are attached to the hands and a cap to monitor upper limb and head movement). The motion sensor nodes capture any movements made by the patient and this information is transmitted back to the base station. The base station constantly receives information from all body sensor nodes and passes this information to a middleware component which filters the data and converts it to 3D data serialisations. Using language optimisations the translated data is used by game components to control the motion of the avatar.

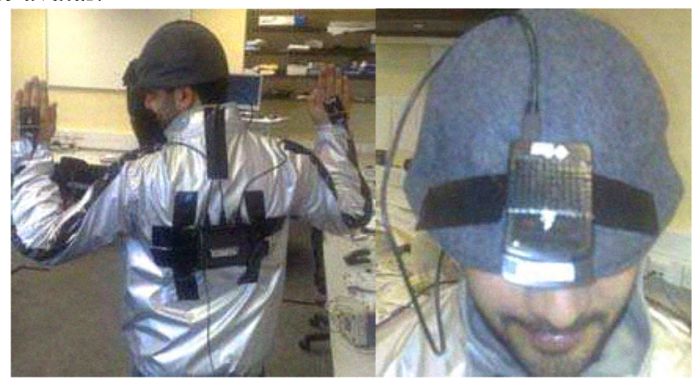

Fig. 3 Body Area WSN during lab experiments

All sensor nodes on the patient's body are used to form a joy stick which allows the patient to control the game avatar by moving their body parts. All body sensors have different communication channels which are directly linked to the base station via radiogram or radio stream connections. Sensors on the body form relationships with other nodes and services within the network. This could be on the request of a plug-in application, such as those provided by a visualisation tool or another framework.

\section{B. Dynamic Gaming (Component two)}

Computer games are a well known source of entertainment and the industry is huge. In the proposed framework we build on the entertainment value gaming provides to help improve the health of patients with disabilities. A by-product of being entertained is that sensor data allows different exercise patterns for different patients to be discovered and used in rehabilitation. This data can then be used by physicians to tailor therapies. Under normal patient physician relations, rehabilitation is purely subjective with no quantitative data usage. Using gaming technologies and the sensor data allows physiotherapy to be formalised and quantitatively evaluated against past and ongoing treatments. Having informative information that can be enacted via the gaming environment allows physicians to help patients in therapy or recommend different exercises for physical health 
improvement.

During the game the framework collects medical and motion information from the patient and uses this information to make decisions about game play. Changes to the game can be subtle to allow for maximum physiotherapy, through body motion, in order to help maximise physical health improvement. For example, the game may require the patient to stretch their arms over their head to collect an object in the game in order to progress to the next level. Here the patient does this to advance the game for the purpose of entertainment and at the same time indirectly performs an exercise to improve aspects of their physical condition. This process helps to improve the patient's health step by step. As all information from the patient is stored it is relatively easy to check the rate of patient health improvement.

\section{Data Acquisition Manager (Component three)}

Every game collects data from the beginning of the interaction until the user completes the game. The data acquisition manager uses this data to generate different reports and graphs to answer doctor and patient queries. Furthermore, this data is used by doctors to replay patient body part motion to check their physical performance in terms of physical appearance or use arithmetic data for exercise accuracy. The data acquisition manager also provides an easy and quick comparison of different games played by patients. The proposed framework is not limited to the improvement of patient health but can also be used as a research tool by physicians and doctors from different areas of medicine. The data source itself can be connected to central hospitals or remote sites to monitor the patient rehabilitation process and physicians can directly communicate with patients using telemedicine equipment.

\section{FRAMEWORK IMPLEMENTATION}

\section{A. Implementation of body area WSN}

To establish a WSN the Sun Microsystems newly developed sensors called SunSPOT have been used. There are two types of SunSPOT; one is called the base station and other is called a free range SPOT. The base station is directly connected with a computer or laptop allowing data to be collected from the WSN. All free range SunSPOTs are attached to the patients body, as shown previously in Figure 3, and preloaded with algorithms to collect acceleration (motion) values (which includes accX, accY and accZ) and send the data to the base station using different communication ports. The base station is connected directly to the computer and runs algorithms that wirelessly collects data from all free range SPOTs. Furthermore, all received data is sent to the middleware for filtering and used by the game component.

Framework services are predetermined, and each device understands how to discover and invoke them. Bindings between devices and framework services (discovery, service matching, and advertisement management) have been developed in Java and connections are established using predefined pipe advertisements. All services redundantly coexist. Sensors that do not explicitly implement framework services can discover and use the services provided by other network nodes. For example, better capable devices may be used to perform computationally expensive functions.

\section{$B$. Implementation of the Visualisation and Game Engine}

A simple 3D biped has been created using several "capsule" geometric instances to represent the limbs and torso of the human user whose motion is being captured (Java Monkey Engine was used). The capsules are stored in Dynamic Physics Nodes, giving them real-time physical properties such as model bound constraints, acceleration under gravity and centre of mass. These nodes are chained together using joints, to allow them to pivot freely. Finally five control joints and nodes are set up to take positional data from the sensors, and pinned to the 3D world using local anchor points to the hands, feet and neck of the biped with a further joint for each one. The torso is anchored to a static/absolute world position with a control node. We have successfully linked SunSPOTs with a virtual representation in a gaming environment. In one example the accelerometer data is used to move a representation of itself in the virtual environment in relation to real-world movements. Obviously there is still a need to create the game play for specific medical conditions, such as obesity, stroke or cerebral palsy.

\section{Data Acqisition Manager}

Currently we are using a simple Microsoft Access database for data storage and JDBC to establish connectivity between the database and the middleware. The data acquisition manager can use the same data to produce many useful results.

What makes the proposed framework novel is the generic platform for sensor and service integration. Potentially any functionality could be integrated into any number of different types of sensor network. Following the protocol rules, all functions can be abstracted as services for use by any other sensor or service in the network. Whilst the focus is on the rehabilitation of debilitating illnesses through body area sensor networks any other conceivable application could be created.

\section{Evaluation}

The proposed framework has been evaluated using a neck therapy case study (side flexions). Neck therapy is a treatment used for neck pain, stiffness and injury. Neck pain is a very common condition and is more frequently seen in women than men. If the pain has lasted more than three months, it is termed 'chronic' neck pain. The most effective physiotherapy techniques for relief from neck pain are neck extension, rotation, side flexions, neck retraction (chicken tuck), upper neck nodding, shoulder shrugs and shoulder bracing (retraction). In this paper we have only shown the results for side flexions.

In side flexion therapy the patient has to tilt their left ear down towards the left shoulder and gently assist this motion and stop when a stretch is felt in the muscles on the right side of the neck. This position is held for a count of ten and repeated in the opposite direction. In side flexion a normal person cannot tilt his/her neck more then 45 degree in both left and right directions as show Figure 4. 
Therefore we have monitored neck motion in between -45 and 45 degrees during experimental therapies sessions.

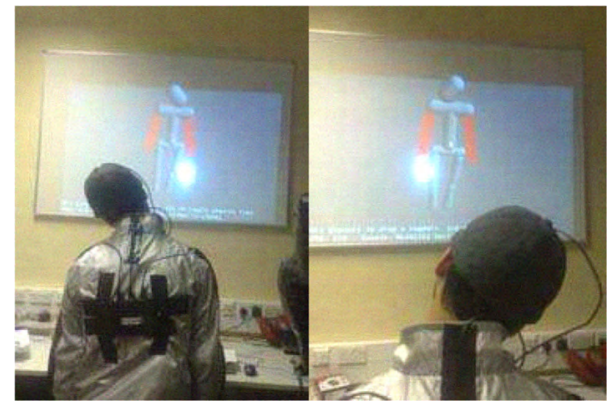

Fig. 4 Neck physical therapy for interior neck muscle

In this case study we attached a sensor node on top of a cap fitted to the head as shown in Figure 3. The patient has to wear the cap and follow the side flexion therapy instructions (mention above) for exercises shown in Figure 4. The results from two different side flexion therapy sessions have been collected and shown in Figure 5 and Figure 6. The neck position is measured every millisecond with the help of the sensors attached to the cap.

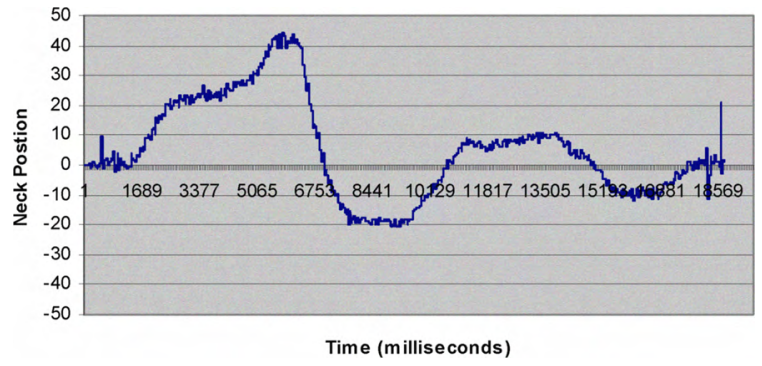

Fig. 5 Side flexion neck therapy session 1

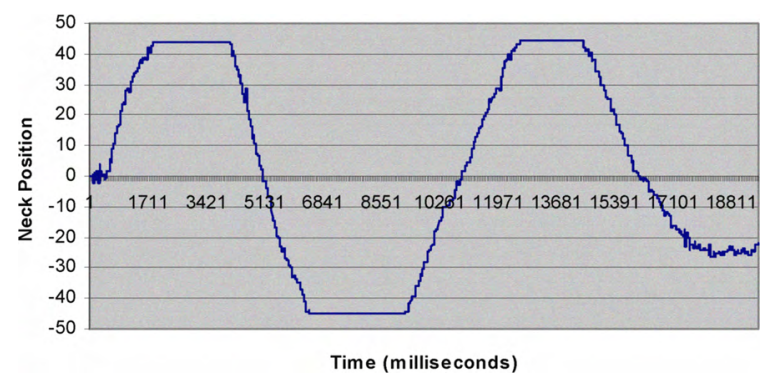

Fig. 6 Side flexion neck therapy session 2

All sensed data values from the sensors are converted into 3D coordinate values, which are then used to animate the 3D model. Figure 5 and Figure 6 demonstrates the total time taken in a specific therapy session; the speed in which the neck was moved; and the position of the neck at different time intervals. In Figure 5 and 6 graphs show the number of curves which in turn represent the neck tilt left and right. Using these values a physician can easily determine how accurate the therapy has been performed. Therapy session 2 (Figure 6) shows that the side flexion is performed more accurately then therapy session 1 (Figure 5). Each of these graphs can be overlaid on top of each other to see the level of improvement and used to tailor future therapy techniques in order to make compensatory changes.

All data in both cases is saved and this helps to record every single therapy session performed by the patient. In a current community setting there is no physical data collection only the subjective opinions and assessments made by the physician. Therefore our novel solution can improve current physiotherapy techniques.

\section{CONCLUSION AND FUTUREWORKS}

In this paper we have presented a novel framework for physical health improvement. The proposed system can be used in-home and within a community setting that incorporates medical installations, such as hospitals. It has three components; the body area WSN, the game environment and the data acquisition manager. A neck physiotherapy case study was used to illustrate the applicability of our approach.

Our project is a work in progress and much still remains to be done. In particular, we hope to run more comprehensive tests in a community setting to provide a better evaluation. We need to incorporate game play to target specific therapies.

\section{REFRENCES}

[1] H. Zhou, T. Stone, H. Hu, and N. Harris, Use of multiple wearable inertial sensors in upper limb motion tracking. Medical Engineering \& Physics, 2008, 30(1): p. 123-133.

[2] D. Wade and B. d. Jong, Recent advances in rehabilitation. Br Med J, Year, p. 1385-1388.

J. Cauraugh and S. Kim, Two coupled motor recovery protocols are better than one electromyogram-triggered neuromuscular stimulation and bilateral movements. Stroke, Year, p. 1589-1594.

[4] G. Burdea, V. Popescu, V. Hentz, and K. Colbert, Virtual reality-based orthopedic telerehabilitation, IEEE Transactions on Rehabilitation Engineering 2000, 8(3): p. 430-432.

[5] A. Milenkovic, C. Otto, and E. Jovanov, Wireless sensor networks for personal health monitoring: Issue and implementation. Computer Communication, 2006, 29: p. 2521-2533.

[6] M. Buckland, B. Frost, and A. Reeves, Liverpool Telecare Pilot: Telecare as an Information Tool. Informatics in Primary Care, 2006, 14(3): p. 191-196.

[7] T. L. Hayes, P. Pavel, N. Larimer, I. A. Tsay, J. Nutt, and A. G. Dami, Distributed Healthcare: Simultaneous Assessment of Multiple Individuals. IEEE Pervasive Computing, 2007, 6(1): p. 36-43.

[8] J. Finkelstein, M. R. Cabrera, and G. Hripcsak, InternetBased Home Astham Telemonitoring: Can patients handle the technology, Chest, 2000, 117(1): p. 148-155.

[9] N. Hosker, Exploiting the potential of Informatics in Health care, Nurse Prescribing, 2007, 4(9): p. 391394. 